Special Issue: Social Cognition and Self. Citation: Dragland, L. (2015). A review of the role of mindfulness-based interventions in the treatment of addiction. Behavioural Sciences Undergraduate Journal, 2(1), 13-23.

Author: Lindsay Dragland, Psychology, Mount Royal University (MRU)

Correspondence: ldrag413@mtroyal.ca

Reviewers: Alain Morin, MRU; Kelsie Moore, MRU; Mabel Yu, MRU

Editor: Famira Racy, MRU

Acknowledgements: The BSUJ acknowledges Dr. Dave Mumby from Concordia University for associate editorial contributions to this article.

Copyright: (c) 2015 Lindsay Dragland. This is an open-access article distributed under the terms of the Creative Commons Attribution 4.0 International License. The use, distribution or reproduction of this article in other forums is permitted, provided the original author(s) or licensor are credited, a link to the licence is provided (CC for 4.0), it is indicated whether or not changes were made to the original article, and other conditions of the BSUJ and the other forums are met.

\title{
A Review of The Role of Mindfulness-based Interventions in the Treatment of Addiction
}

\begin{abstract}
Mindfulness involves the nonjudgmental awareness and observation of one's feelings, thoughts, and behaviours. Mindfulness-based interventions are becoming increasingly popular, as the growing body of research suggests promising implications regarding mindfulness as an approach to treat a wide variety of health problems. Specifically, mindfulness practices have been utilized in the treatment of addiction, and are shown to significantly reduce the relapse rate for individuals struggling with addiction. This paper reviews the research on mindfulness and addiction, describing the conceptualization and measurement of mindfulness, as well as various mindfulness-based interventions. An overview of addiction, including its determinants and treatments, is also provided. The relationship between mindfulness and addiction is examined and conclusions are drawn about the effectiveness of mindfulness interventions, how these interventions address the essential components of recovery, and the extent to which mindfulness may be utilized as a preventative measure.
\end{abstract}

Keywords: addiction, alcohol use, cognitive therapy, genetic predisposition, meditation, mindfulness, relapse, self-awareness, self-knowledge, stress reduction, substance use

\section{Introduction}

Addiction is a chronic, relapsing disorder that has become a global epidemic contributing to millions of deaths per year, and drastically lowering the quality of life for several million individuals worldwide (Brandon, Vidrine, \& Litvin, 2007). Therefore, it is important to understand the various approaches to treating addiction, and what can be done to prevent the numerous problems associated with this detrimental disorder. Cognitive-behavioural therapies have increasingly been incorporating mindfulness approaches as strategies to treat a variety of disorders and problems (Keng, Smoski \& Robins, 2011). Specifically, mindfulness has been shown to produce promising outcomes for addicted individuals in the process of recovery (Brewer et al., 2009; Bowen, Chawla \& Marlatt, 2011). The efficacy of mindfulness approaches to recovery is largely attributed to decreasing the impact of negative affect, or subjective, 
unpleasant, and aversive feelings or emotions such as anxiety and sadness (Kassel, Veilleux, Wardle, Yates, Greenstein, Evatt \& Roesch, 2007).

Negative affect is a well known trigger for substance use relapse (Khanna \& Greeson, 2013). In what follows, I will examine the ways in which mindfulness-based interventions can be used as effective approaches to treating addiction. In doing so, I will begin with an overview of mindfulness-- including the different types of mindfulness, and the ways in which mindfulness has been measured. Next, I will provide an overview of addiction, describe the determinants of addiction, and mention various established treatments for addiction. Lastly, I will explain the relationship between mindfulness and addiction, by providing a description of the various mindfulness-based interventions and how they have been implemented in the treatment of addiction.

\section{Mindfulness}

Broadly speaking, mindfulness has been defined as non-elaborative, non-evaluative awareness and observation of one's feelings, thoughts, and behaviours as they occur, for example simply noticing and accepting one's own feelings without speculating about their causes or effects (Carlson, 2013). Mainly rooted in the ancient spiritual tradition of Buddhism, mindfulness first began to integrate into Western medicine and psychology in the 1950s and 1960s with the expansion of Zen Buddhism in America (Keng et al., 2011). However, it was over a decade after its integration that mindfulness meditation began to be studied as an intervention to improve psychological wellbeing because of its positive association with components of wellbeing such as purpose in life, positive emotions, meaningful relationships, and sense of accomplishment (Slavin, Schindler, Chibnall, Fendell \& Shoss, 2012).

A general relationship between mindfulness and wellbeing has been widely supported both theoretically and empirically, but specific elements of mindfulness such as nonjudgmental selfawareness and self-acceptance seem to be related to overcoming maladaptive coping tendencies (Keng et al., 2011). Such maladaptive tendencies include avoidance, or escape and selfdistraction, suppression, or ignoring and denying, and rumination, or excessive self-focus involving anxious, persistent, repetitive, generic questioning of negatively perceived self-aspects (Baer, 2003). Mindfulness interventions specifically teach individuals to notice and accept their cognitive reactions to emotions, which is related to improving self-awareness and targeting of rumination and facilitating the reduction of negative thought patterns and rumination (Caselli, Gemelli, Querci, Lugli, Canfora, Annovi, \& Watkins, 2013). In other words, reduction of maladaptive coping is related to increased wellbeing, and mindfulness can facilitate this process.

\subsection{Conceptualization and Measurement}

In a thorough review of the literature on mindfulness and self-knowledge, Carlson (2003) found that mindfulness can be conceptualized and assessed as a trait, a state, or a trained skill. Trait mindfulness refers to an individual's capacity and tendency to be mindful in everyday life; it is assessed with self report measures and can be increased with training, although training is not necessary for one to be mindful. State mindfulness, on the other hand, refers to an individual's momentary episode of mindfulness, rather than one's general inclination to be mindful. Self report measures are used to assess state mindfulness as well, but assessment is done after state mindfulness is momentarily induced, often with a brief mindfulness exercise such as guided meditation. The concept of mindfulness as a skill refers to one's mindfulness abilities, acquired by the practice of mindfulness meditation or non-elaborative observation of 
constantly changing internal stimuli as they arise (Baer, 2003). This skill can be learned through mindfulness-based courses, mindfulness training and exercises, or mindfulness-based clinical interventions, and assessed with self-report measures (Carlson, 2013).

Findings of positive associations between self-report mindfulness inventories and reports of wellbeing, as well as the reduction of maladaptive coping tendencies and reported defensiveness is support theoretical construct validity of these self-report measures. (for psychometric properties of related measures see Hsu, Collins, \& Marlatt, 2013).

\subsection{Empirical Evidence}

Research findings indicate that mindfulness can be measured and identified at not only subjective levels, but neurobiological and behavioural levels as well. There have been many subjective measures developed to assess mindfulness. Specific self report questionnaires assessing trait mindfulness include the Freiburg Mindfulness Inventory, Kentucky Inventory of Mindfulness Skills, Mindful Attention Awareness Scale, Five-Facet Mindfulness Questionnaire, and Cognitive Affective Mindfulness Scale (Keng et al., 2011). Findings from self-report measures show an association between trait mindfulness and many elements of wellbeing such as higher levels of life satisfaction, conscientiousness, self esteem, empathy, competence, optimism, and pleasant affect (Keng et al., 2011) as well as lower levels of mind-wandering and defensiveness to ego-threatening information (Carlson, 2013).

Neuroimaging techniques have also been successfully used as mindfulness measures, and have found structural and functional differences in the brains of mindful individuals compared to individuals not trained in mindfulness. For instance, with the use of functional magnetic resonance imaging (fMRI), Hölzel and colleagues found that mindfulness meditation experience was associated with increased activation in two brain areas involved in emotional processing (the dorsal medial prefrontal cortex) and attentional control (the rostral anterior cingulate cortex; Hölzel, Ott, Hempel, Hackl, Wolf, Stark \& Vaitl, 2007). At the behavioural level, various performance-based measures have shown that mindfulness training is associated with enhanced cognitive flexibility and attentional functioning. For instance, it was found that those who had undergone mindfulness training demonstrated superior executive attention skills compared to untrained individuals (Carlson, 2013). The fact that mindfulness levels can be assessed in terms of both subjective and objective measures paves the way for further empirical research assessing mindfulness as a significant variable in improving the human condition.

\subsection{Mindfulness-based Interventions}

The goal of mindfulness-based interventions is to evoke greater reflection and acceptance through the development of a nonjudgmental awareness, curiosity, openness, and acceptance of external and internal experiences--especially with regard to negative affect (de Dios, Herman, Britton, Hagerty, Anderson \& Stein, 2012). Mindfulness-based interventions for the treatment of clinical problems began with the work of Jon Kabat-Zinn, which applied mindfulness meditation to the treatment of chronic pain--a treatment now widely known as Mindfulness-Based Stress Reduction (MBSR; Keng et al. 2011; for more on this method see Kabat-Zin \& Hanh, 2009).

After the emergence of MBSR, several other mindfulness-based interventions were developed and applied to the treatment of a variety of problems, with promising results. Such interventions include Mindfulness-Based Cognitive Therapy (MBCT), Acceptance and Commitment Therapy (ACT), Mindfulness-based relapse prevention (MBRP), and Mindfulness Oriented Recovery Enhancement (MORE). I will explain these various approaches in detail later 
on when I describe how they have been applied to the treatment of addiction. For now though, it is important to understand just what addiction entails, and what factors have contributed to its development and relapse rates.

\section{Substance Abuse, Dependence and Addiction}

Although the terms substance 'abuse' and 'dependence' are often used interchangeably with the term 'addiction,' the three are not synonymous. I will begin by defining these terms and highlighting their differences for clarification; but for the sake of this paper, I will be referring to them overall as addiction, because abuse and dependence often accompany addiction. Substance abuse relates to the problematic use of psychoactive substances (alcohol, and both prescription and illicit drugs) and can refer to anything from occasional to repeated use. Continued use of such substances may induce changes in the central nervous system that can lead to physical dependence-- a condition in which the body adapts to the substance, resulting in increased tolerance and physical or mental symptoms of withdrawal if substance use is abruptly ceased (Camí \& Farré, 2003).

Depending on the substance being abused, physical dependence usually accompanies addiction-- a chronic, relapsing disorder characterized by a compulsive pattern of drug-seeking and drug-taking behaviour that persists despite negative consequences, such as failure to meet work, social, or family obligations (Robinson \& Berridge, 2003). Around 27 million people worldwide suffer from severe drug problems; over five percent of the global burden of disease is attributable to alcohol and illicit drug use, and alcohol abuse accounts for about 2.5 million deaths per year (Brandon et al., 2007). Therefore, it is important to understand the various approaches to treating addiction, and what can be done to prevent the numerous problems associated with addiction.

\subsection{Causes of Addiction}

Most would agree that addictive substances induce pleasant states or relieve unpleasant ones (Camí \& Farré, 2003), and that addiction often starts with the addict taking a substance chiefly for pleasure or to relieve distress (Robinson \& Berridge, 2003). However, there are also many other factors that contribute to the risk of addiction including withdrawal, cognitive deficits, personality traits, psychiatric disorders, and genetics, shown below.

\subsubsection{The Pleasure-Withdrawal Theory}

The basic logic behind the traditional pleasure-withdrawal view is that addictive substances are taken initially simply to achieve a pleasant state, but with repeated use comes tolerance and dependence, leading to withdrawal when substance use is ceased. As such, compulsive drug taking is maintained with attempts to avoid or relieve unpleasant withdrawal symptoms (Robinson \& Berridge, 2003). While this notion is the most intuitive explanation for addiction, it is a known fact that those with addictions often start abusing substances again, even long after they become free from unpleasant withdrawal symptoms. Therefore, elimination of such symptoms does not eliminate the risk of relapse, and other factors must be involved in addiction.

\subsubsection{The Incentive-Sensitization Theory}

Those with addictions often continue to abuse substances despite being aware of all the problems that their substance use is causing, so the motivation to abuse such substances must be a force much stronger than mere liking. Repeated use of addictive substances can lead to 
dissociation between the liking of a substance and the wanting of a substance, which is why an alcoholic may no longer even like alcohol, yet still have intense cravings for alcohol. The incentive-sensitization theory posits that the brain's reward mechanisms can become hypersensitized to both the direct effects of a substance and the stimuli that are not directly associated with the substance (Camí \& Farré, 2003). In other words, incentive sensitization can be defined as an intense pathological wanting or craving that persists even though liking progressively decreases-- a dissociation that develops because the brain become sensitized to the substance after repeated use.

\subsubsection{Genetic Factors}

In their comprehensive review of the literature on drug addiction, Camí and Farré (2003) found that the risk of addiction may increase or decrease depending on genetic factors that influence the metabolism and the effects of substances; specifically, findings from numerous family and gene studies indicate that genetic predisposition plays a large role in the development of addiction. For instance, findings from various twin studies indicate a high probability of being addicted to alcohol when one's identical twin is addicted to alcohol (Camí \& Farré, 2003). Other family studies indicate that men who had alcoholic parents have a reduced sensitivity to alcohol, a factor associated with the development of alcoholism; furthermore, the likelihood of alcoholism increased among these men, despite being adopted at birth and raised by nonalcoholic parents (Schuckit et al., 2001).

Other studies analyzing allelic variants indicate that those with an allele of aldehyde dehydrogenase are less likely to abuse alcohol because of increased levels of acetaldehyde, a chemical compound responsible for aversive effects from alcohol consumption (Nestler, 2000). Further analysis also found that the minor (A1) allele of the Taq IA D2 dopamine receptor gene is associated with severe alcoholism, polysubstance and psychostimulant abuse or dependence, and opioid and nicotine dependence (Noble, 2003).

\subsubsection{Personality Traits and Psychiatric Disorders}

Personality traits and psychiatric disorders are significant contributing factors associated with addiction. Compulsive drug-seeking and drug-taking behaviour has been shown to be facilitated by risk-taking or novelty-seeking personality traits, as well as cognitive deficits such as difficulties in decision making and evaluating the consequences of one's behaviour (Camí \& Farré, 2003). Alcohol relapse is associated with cognitive, emotional, and behavioural mechanisms including attentional bias toward alcohol-related stimuli, thought suppression, poor emotion regulation skills, and emotional reactivity leading to compulsive drug-seeking behaviour; negative affect and perceived stress have also been shown to increase the relapse risk for both alcohol and cigarette use (Khanna \& Greeson, 2013). Numerous psychiatric disorders, especially schizophrenia, bipolar disorder, depression, and attention-deficit-hyperactivity disorder, increase the risk of addiction. Consequently, investigating the comorbidity of addiction and psychiatric disorders is crucial, as a dual diagnosis has unfavourable implications for management and outcome (Camí \& Farré, 2003).

\subsection{Approaches to Treating Addiction}

The concept of addiction as a chronic, relapsing disorder is fundamental to our understanding of recovery and how to go about treating addiction. Recovery requires more than mere abstinence from addictive substances; as such, according to Kelly and White (2011), many 
have embraced the concept of recovery as a process of change through which an individual achieves not only abstinence, but also improved health, wellness, and quality of life. With the notion of recovery being more broadly defined, the focus has shifted from pathology to more positive outcomes, and also has progressed toward embracing long-term and continuity-of-care models for understanding and treating addiction (Kelly \& White, 2011).

There are a wide variety of treatment programs focusing on recovery that individuals suffering from addiction may choose from (for examples of recovery management in treatment practice, see Boyle, Loveland \& George, 2011; Valentine, 2011). Medical therapy for withdrawal symptoms, residential therapeutic communities, self-help groups, hospital-based inpatient programs, and drug-free outpatient programs are available and provide a combination of services that vary in quality and quantity (e.g., individual, group, and family counseling, cognitive-behavioural therapy, relapse prevention training, medical care, educational and occupational training, and social and community support; Hser \& Angelin, 2011).

\subsubsection{Cognitive-Behavioural Relapse Prevention}

Prevailing interventions for relapse prevention aim to help individuals through the process of recovery by attending to their cognitive, behavioural, and lifestyle choices that may be changed or promoted to help avoid relapse (Hser \& Angelin, 2011). Such cognitive-behavioural interventions focus on addressing various determinants of relapse, including physical withdrawal symptoms, cognitive processes such as craving, self efficacy, outcome expectancies, motivation, adaptive and maladaptive ways of coping, emotional states and self-regulating negative affect, and situational or contextual factors that elicit craving (Khanna \& Greeson, 2013). Ultimately, what is central to this approach is helping individuals identify relapse triggers and strengthen their overall coping capacity to cognitively deal with these triggers and avoid relapse.

\section{The Connection between Mindfulness, Addiction and Treatment}

In many ways, addiction is best understood as a maladaptive response to distressing states of known and unknown origin. Many recovering addicts continue to relapse time and time again because of uncomfortable internal experiences that arise, which the individual assumes are intolerable and consequently seeks immediate relief through substance use (Robinson \& Berridge, 2003). Mindfulness can help such individuals become aware of, safely explore, and be less troubled by these distressing experiences, so that alternative, adaptive ways of responding can be employed (Keng et al., 2011).

\subsection{Mindfulness-based Approaches to Addiction}

For many, a major challenge to overcoming addiction is the belief that changing one's maladaptive coping habits is near impossible, as many addicts view their internal experiences, such as thoughts and emotions, as facts that are a part of their essence (Keng et al., 2011). Mindfulness practices help individuals disengage from these experiences, realize that they are not their thoughts, and view their thoughts as mental events rather than as facts; consequently, individuals will begin to exercise more positive ways of looking at and dealing with their distressing internal experiences, and realize that positive change is achievable (de Dios et al., 2012). 


\subsection{Mindfulness-Based Stress Reduction (MBSR)}

Mindfulness-Based Stress Reduction (MBSR) is a group-based intervention that offers intensive training in mindfulness meditation to help patients with chronic pain reflect on their physical and psychological conditions in a more accepting and nonjudgmental manner. This eight to ten week program consists of weekly in-class mindfulness meditation instruction and training; patients are also encouraged to practice their mindfulness exercises at home, as well as attend an all-day mindfulness meditation retreat (Keng et al., 2011). Because MBSR utilizes intense and continual training in mindfulness meditation, the idea is that participants will, in due time, break away from their maladaptive thinking and behaviour, and learn more accepting and nonjudgmental ways to relate to their experiences.

It is evident that stress increases the likelihood of substance use and can trigger relapse following recovery (Khanna \& Greeson, 2013). Therefore, there is a crucial need to incorporate stress management techniques into inpatient and outpatient treatment programs for addiction. Stressful life circumstances are most likely to prompt substance use among impulsive individuals with maladaptive coping behaviours, and those who are motivated to avoid negative affect (Kelly \& White, 2011). These individuals are known to use substances as a form of avoidance coping that involves self-medicating to reduce stress and depression; such maladaptive coping, if successful, reinforces substance use (Kelly \& White, 2011). Therefore, because MBSR helps individuals break free from their maladaptive habits, and learn to positively reflect on and accept their experiences, the idea is that substance use will be replaced with adaptive coping skills when individuals are confronted with the inevitable life stressors that threaten recovery.

\subsection{Mindfulness-based Cognitive Therapy (MBCT)}

Adapted from the MSBR model, Mindfulness-Based Cognitive Therapy (MBCT) is a preventative treatment aimed at reducing the risk of relapse in recurrent depression by changing an individual's awareness of and relationship to thoughts and emotions. This eight week groupbased program combines mindfulness training and elements of cognitive therapy to help individuals view their thoughts as mental events, rather than as facts, and recognize the role that their negative automatic thoughts have in perpetuating depressive symptoms (i.e., cognitive process of learning oneself is not one's thoughts and thoughts can be changed; Kabat-Zinn \& Hanh, 2009).

Because MBCT targets mental processes involved in the maintenance of depressive episodes, the idea is that the amount of these episodes, as well as the risk of relapse, will be decreased with treatment. MBCT is similar to MBSR in dealing with addiction and relapse prevention. If individuals can develop a better awareness of, and way of coping with, negative affect, thoughts, and emotions that are often associated with stress and depression, then the risk of relapse for these individuals will greatly decrease (Keng et al., 2011).

\subsection{Acceptance and Commitment Therapy (ACT)}

Unlike most mindfulness-based interventions, Acceptance and Commitment Therapy (ACT) does not incorporate the practice of mindfulness meditation; it does, however, stick with the overall goal of such interventions--to evoke greater awareness and acceptance of the self (Dermatis \& Egelko, 2014). ACT employs a variety of exercises directed at cultivating the deliteralization of experiences by observing the self with a detached attitude toward internal and external phenomena. The program can last anywhere from one day to sixteen weeks, and has been administered in both individual and group settings (Keng et al., 2011). Six core treatment 
processes are involved (i.e., acceptance, diffusion, contact with the present moment, self as context, values, and committed action)-- the first four of which respectively incorporate mindfulness training.

The central aim of ACT is to decrease an individual's tendency to avoid negative internal events, and increase the tendency to engage in behaviours that are consistent with their values and goals (Keng et al., 2011; Dermatis \& Egelko, 2014). Therefore, ACT can effectively help recovering addicts decrease the tendency to use substances as a form of avoidance coping, and increase the tendency to behave in ways that are consistent with their goal of successful recovery. Keng and colleagues (2011) found that several studies show ACT is an effective intervention for treating a range of other psychological problems including depression, anxiety, impulse control disorders, and schizophrenia, all of which have been linked to substance use and an increased risk for relapse.

\subsection{Mindfulness-Based Relapse Prevention (MBRP)}

Mindfulness-based relapse prevention (MBRP) is a group-based, psychoeducational intervention for the treatment of substance use disorders. This eight week outpatient program is a combination of traditional cognitive-behavioural relapse prevention strategies and mindfulness meditation training (Khanna \& Greeson, 2013). Like most mindfulness techniques, MBRP aims to help individuals develop awareness and acceptance of internal events-- especially those involving substance craving-- and to develop effective coping strategies that can be utilized when faced with high-risk situations, especially when experiencing negative affect (Hsu, Collins \& Marlatt, 2013). For example, some MBRP programs have included sessions that specifically target working with anger as a trigger for stress or drug use, and using loving-kindness techniques to facilitate attending to and dealing with difficult emotions (Brewer et al., 2009).

\subsection{Mindfulness Oriented Recovery Enhancement (MORE)}

Mindfulness Oriented Recovery Enhancement (MORE) is adapted from the MBCT model for depression treatment, and utilizes many of the same mindfulness meditation practices that have been included in MBCT, MBSR, and MBRP (Khanna \& Greeson, 2013). This ten session, group-based, psychoeducational intervention is aimed at treating substance dependence and other forms of addiction. MORE implements meditative approaches to coping with cravings, and provides education and training about mindfully being aware of, observing, and skillfully changing psychological mechanisms implicated in addiction and dependence (Khanna \& Greeson, 2013).

Recovery enhancement can be achieved through the application of informal mindfulness practices in everyday life, which focus on developing awareness of automatic behaviour in order to reduce mind-wandering and strengthen conscious control over impulsivity; for example, some interventions instruct individuals to engage in tedious, everyday tasks (e.g., doing laundry) with full awareness of the sensorimotor aspects of the task at hand (Garland, Froeliger \& Howard, 2013). Essentially, MORE is designed to target cognitive, affective, and physiological processes that are involved in the maintenance of addictive behaviours and maladaptive ways of thinking.

\section{Future Directions}

Addiction is a global epidemic that claims millions of lives each year; because it is a chronic disorder, many individuals will suffer several episodes of relapse before full recovery, and even more will likely never fully recover from their addiction. Therefore, in terms of future 
research, it would be beneficial to conduct studies on mindfulness as a potential preventative measure which may help impede one's addictive tendencies, and ultimately contribute to the prevention of addiction before it even gets a chance to consume the lives of vulnerable individuals in the first place. Findings from numerous family and twin studies have indicated a strong association between genetics and addiction. For this reason, it may be advantageous for parents who have suffered from addiction to take precaution and begin implementing mindfulness meditation and training practices into not only their own lives, but their children's lives as well. Consequently, if parents know that their child may have a genetic predisposition for substance use and addiction, it would be beneficial for them to also know whether engaging their child in mindfulness practices early on can help decrease the likelihood of that child adopting her parents' addictive tendencies. As a result, we may be one step closer to stopping the global epidemic of addiction in its tracks.

While mindfulness as a means to prevent addiction sounds promising, it is-- to say the least-- a highly optimistic notion. Mindfulness interventions may address some cognitive, emotional, and behavioural aspects associated with addiction (e.g., thought suppression, poor emotional regulation and emotional reactivity; Khanna \& Greeson, 2013), but mindfulness alone cannot address the more complex factors associated with addiction such as genetic predispositions (e.g., individual differences in acetaldehyde levels), psychiatric disorders (e.g., schizophrenia), and personality traits (e.g., risk-taking and novelty-seeking traits; Camí \& Farré, 2003). Therefore, while mindfulness is a highly promising practice that has been show to increase the likelihood of successful recovery, future research may benefit by further exploring mindfulness in conjunction with other practices (e.g., medical therapy, inpatient programs, social and community support; Kelly \& White, 2011) as a means to recover from, and possibly even prevent addiction.

\section{Conclusion}

Mindfulness involves awareness, reflection and acceptance of both positive and negative experiences, and is ultimately aimed at creating a life worth living (Corey, 2013). Mindfulnessbased approaches for addiction treatment focus on improving distress tolerance and coping skills, and help to eliminate maladaptive, automatic avoidance of negative affect and consequential substance use (de Dios et al., 2012). With the concept of addiction as a relapsing disorder, comes the concept of recovery as a process of change through which an individual achieves not only abstinence, but also improved health, wellness, and quality of life (Kelly \& White, 2011). Because mindfulness-based interventions centre on practices that effectively improve psychological well being and overall quality of life, not only do these interventions specifically address the essential components of recovery, they also embrace the long-term and continuity-ofcare models which have been increasingly crucial for understanding and treating addiction. Therefore, further research must be done on the relationship between mindfulness and addiction, and the various ways in which mindfulness interventions can be used in conjunction with existing addiction treatments to increase the likelihood of successful recovery.

Mindfulness can be operationalized, conceptualized and assessed as a trait, a state, or a trained skill (Carlson, 2003); this is a huge strength for mindfulness, as the empirical evaluation of an intervention is of extremely high regard, and requires clear operational definitions and conceptually sound mechanisms (Baer, 2003). However, while mindfulness-based interventions can be rigorously operationalized, conceptualized, and empirically evaluated, to do so risks neglecting some essential elements of traditional mindfulness practices-- elements such as 
cultivation of awareness, insight, and compassion (Baer, 2003). These essential components are complicated subjective constructs that are difficult to put through methodologically rigorous investigations, thereby complicating the empirical validation of mindfulness-based interventions (Baer, 2003). Therefore, future researchers may benefit from examining the ways in which concepts such as insight and compassion may be empirically assessed and incorporated into the meticulous investigation of mindfulness.

\section{References}

Baer, R. A. (2003). Mindfulness training as a clinical intervention: A conceptual and empirical review. Clinical Psychology: Science and Practice, 10, 125-143.

Bowen, S., Chawla, N., \& Marlatt, G. A. (2011). Mindfulness-based relapse prevention for addictive behaviors: A clinician's guide. New York, NY: Guilford Press.

Brandon, T.H., Vidrine, J.I., \& Litvin, E.B. (2007). Relapse and relapse prevention. Annual Reviews of Clinical Psychology, 3, 257-284.

Boyle, M., Loveland, D., \& George, S. (2011). Implementing recovery management in a treatment organization. In Addiction Recovery Management (pp. 235-258). Humana Press.

Brewer, J. A., Sinha, R., Chen, J. A., Michalsen, R. N., Babuscio, T. A., Nich, C., \& Rounsaville, B. J. (2009). Mindfulness training and stress reactivity in substance abuse: Results from a randomized, controlled stage I pilot study. Substance Abuse, 30, 306-317.

Camí, J., \& Farré, M. (2003). Drug addiction. The New England Journal of Medicine, 349(10), 975-986.

Carlson, E. N. (2013). Overcoming the barriers to self-knowledge: Mindfulness as a path to seeing yourself as you really are. Perspectives on Psychological Science, 8(2), 173-186.

Caselli, G., Gemelli, A., Querci, S., Lugli, A. M., Canfora, F., Annovi, C., \& Watkins, E. R. (2013). The effect of rumination on craving across the continuum of drinking behaviour. Addictive Behaviors, 38(12), 2879-2883.

Corey, G. (2013). Theory and practice of counseling and psychotherapy ( $9^{\text {th }}$ ed.). Scarborough, Ontario: Brooks/Cole.

de Dios, M. A., Herman, D. S., Britton, W. B., Hagerty, C. E., Anderson, B. J., \& Stein, M. D. (2012). Motivational and mindfulness intervention for young adult female marijuana users. Journal of Substance Abuse Treatment, 42(1), 56-64.

Dermatis, H., \& Egelko, S. (2014). Buddhist mindfulness as an influence in recent empirical CBT approaches to addiction: Convergence with the Alcoholics Anonymous model. Alcoholism Treatment Quarterly, 32(2-3), 194-213.

Garland, E. L., Froeliger, B., \& Howard, M. O. (2013). Mindfulness training targets neurocognitive mechanisms of addiction at the attention-appraisal-emotion interface. Frontiers in Psychiatry, 4.

Hölzel, B. K., Ott, U., Hempel, H., Hackl, A., Wolf, K., Stark, R., \& Vaitl, D. (2007). Differential engagement of anterior cingulate and adjacent medial frontal cortex in adept meditators and nonmeditators. Neuroscience Letters, 421(1), 16-21.

Hser, Y-I., \& Angelin, M.D., (2011). Addiction treatment and recovery careers. In Addiction Recovery Management (pp. 9-30). Humana Press.

Hsu, S. H., Collins, S. E., \& Marlatt, G. A. (2013). Examining psychometric properties of distress tolerance and its moderation of mindfulness-based relapse prevention effects on alcohol and other drug use outcomes. Addictive Behaviors, 38(3), 1852-1858.

Kabat-Zinn, J., \& Hanh, T.N. (2009). Full catastrophe living: Using the wisdom of your body and mind to face stress, pain, and illness. Random House LLC.

Kassel, J. D., Veilleux, J. C., Wardle, M. C., Yates, M. C., Greenstein, J. E., Evatt, D. P., \& Roesch, L. L. (2007). Negative affect and addiction. Stress and Addiction: Biological and Psychological Mechanisms, 171-189.

Kelly, J. F., \& White, W. (2011). Recovery management and the future of addiction treatment and recovery in the USA. In Addiction Recovery Management (pp. 303-316). Humana Press. 
Keng, S., Smoski, M. J., \& Robins, C. J. (2011). Effects of mindfulness on psychological health: A review of empirical studies. Clinical Psychology Review, 31(6), 1041-1056.

Khanna, S., \& Greeson, J. M. (2013). A narrative review of yoga and mindfulness as complementary therapies for addiction. Complementary Therapies in Medicine, 21(3), 244-252.

Nestler, E. J. (2000). Genes and addiction. Nature genetics, 26(3), 277-281.

Noble, E. P. (2003). D2 dopamine receptor gene in psychiatric and neurologic disorders and its phenotypes. American Journal of Medical Genetics Part B: Neuropsychiatric Genetics, 116(1), 103-125.

Robinson, T. E., \& Berridge, K. C. (2003). Addiction. Annual Review of Psychology, 54(1), 25-53.

Schuckit, M. A., Edenberg, H. J., Kalmijn, J., Flury, L., Smith, T. L., Reich, T., \& Foroud, T. (2001). A genome wide search for genes that relate to a low level of response to alcohol. Alcoholism: Clinical and Experimental Research, 25(3), 323-329.

Slavin, S. J., Schindler, D., Chibnall, J. T., Fendell, G., \& Shoss, M. (2012). PERMA: A model for institutional leadership and culture change. Academic Medicine, 87(11), 1481.

Valentine, P. (2011). Peer-based recovery support services within a recovery community organization: The CCAR experience. In Addiction Recovery Management (pp. 259-280). Humana Press. 